\title{
A rapid method for determining decarboxylase and dihydrolase activity
}

\author{
K. BROOKS ${ }^{1}$ AND T. SODEMAN \\ From the Department of Pathology, Microbiology Section, University Hospital, \\ Ann Arbor, Michigan, USA
}

SYNOPSIS A total of 764 fresh clinical isolates were used to test a rapid method for determining lysine, arginine, and ornithine decarboxylase activity as well as arginine dihydrolase activity. Theĩ conventional Møller decarboxylase broth was tested in parallel with the rapid method on 234i Enterobacteriaceae and 140 non-fermentative Gram-negative rods. The $0.3 \%$ agar method was tested $\vec{\infty}$ in parallel on 245 Enterobacteriaceae and 146 non-fermentors. All media were checked at half-hour 0 or hourly intervals for up to eight hours, with the final reading taken after incubation for 24 hours ${ }_{-}^{\circ}$ at $37^{\circ} \mathrm{C}$. The rapid method detected 17 positive decarboxylase or dihydrolase reactions that were $\vec{T}$ not detected by the Møller broth and 16 more than the agar medium when testing Enterobacteriaceae. The corresponding figures for the nonfermentative Gram-negative rods were three and two respectively. Lysine and ornithine decarboxylase were generally detected by the rapid broth in two to four hours' incubation while the arginine decarboxylase and dihydrolase were slower and required $\vec{\theta}$ six to eight hours. This compares with overnight incubation as the general rule for the Møller broght $\$$ and agar decarboxylases. The comparable accuracy of the rapid method with conventional teckniques and the shorter incubation time required for detection of positive reactions make this pro cedure well suited to a routine clinical laboratory.

Lysine, arginine, and ornithine decarboxylase tests, as well as the arginine dihydrolase test, are commonly used for identification of Enterobacteriaceae and Aeromonas species (Blair, Lenette, and Truant, 1970; Edwards and Ewing, 1972). More recently, it has been suggested by Gilardi (1971 and 1972) that these tests may also be of some value in identifying various non-fermentative Gram-negative bacilli. Several rapid techniques have been developed to assist the clinical laboratory in testing for these enzymes (Fay and Barry, 1972; Goldschmidt, Lockhart, and Perry, 1971; Richard, 1968). The most promising and simplest of these is described by Fay and Barry (1972) for ornithine decarboxylase.

It is the attempt of this study to determine whether Fay and Barry's test for ornithine decarboxylase can be used in a similar manner to assay for lysine and arginine decarboxylase and arginine dihydrolase. Most other investigators have concentrated on the use of these rapid techniques for

${ }^{1}$ Present address: Mt Sinai Hospital, Bacteriology Laboratory, University Circle, Cleveland, Ohio 44106

Received for publication 20 November 1973. identification of Enterobacteriaceae. The present $\stackrel{2}{\vec{F}}$ study expands the application of this rapid tech- $\frac{}{3}$ nique to include members of the genus Aeromonas as well as non-fermentative gram-negative bacilli.

\section{Materials and Methods}

A total of 764 fresh clinical isolates was used for this study: 479 were Enterobacteriaceae and the remaining 286 were non-fermentative Gram-negative bacilli. All were initially isolated on $5 \%$ sheep blood 윽 agar (BBL) or MacConkey agar plates (BBL), $\frac{D}{2}$ depending on the nutritional requirements of the organisms. The Enterobacteriaceae were identified $\bar{N}$ by the methods of Edwards and Ewing (1972). The non-fermentative, Gram-negative rods were 0 identified according to the scheme in table I. All N organisms were supplied to the experimenter as unknowns.

The base for the decarboxylase test media was made according to the method of Fay and Berry? (1972). It contained $5.0 \mathrm{~g}$ of peptone (Difco), $3.0 \mathrm{~g}$ 뭉 of yeast extract (Difco), and $5 \mathrm{ml}$ of $0.2 \%$ bromocresol purple in $50 \%$ ethanol in $1000 \mathrm{ml}$ of distilled 


\begin{tabular}{ll}
\hline Biochemical Test & Reagent of Manufacturer \\
\hline Oxidase & $1 \%$ tetramethyl-p-phenylene-diamine-dihydrochloride \\
Motility & Motility sulphide (BBL)-prepared \\
Simmons citrate & BBL-prepared \\
P agar & Pseudomonas Agar P (Difco) dehydrated \\
Nitrate & KNO3 (BBL) prepared \\
Growth at $42^{\circ} \mathrm{C}$ & BHI slant (BBL-prepared) in $42^{\circ}$ C waterbath \\
$1 \%$ of Glucose & Phenol red indicator (Hyland) prepared \\
$1 \%$ of Maltose & Phenol red indicator (Hyland) prepared \\
$1 \%$ of Mannitol & Phenol red indicator (Hyland) prepared \\
$1 \%$ of Xylose & Phenol red indicator (Hyland) prepared \\
Lecithinase & TSA (BBL) dehydrated with 10\% egg yolk suspension \\
DNAse & DNAse test Agar (Difco) dehydrated. No indicator. Flood in with 1 N HCL \\
BHI broth-slide motility & BHI broth (BBL) prepared \\
\hline
\end{tabular}

Table I Biochemical scheme for identification of non-fermentative Gram-negative bacteria ${ }^{1}$

${ }^{1}$ If organisms cannot be identified with the initial eight biochemical reactions, then the later five are inoculated. If an identification still cannot be made, additional biochemical tests may be employed. For information on interpretation of results the reader is referred to Gilardi.

water. The solution was heated gently until all additives were completely dissolved. Then $10 \mathrm{~g}$ of the appropriate amino acid (lysine, arginine, or ornithine) was added and the $\mathrm{pH}$ of the solution adjusted to $5 \cdot 0-5 \cdot 5$ with $\mathrm{HCl}$. One millilitre aliquots of the broth were tubed and autoclaved. All rapid decarboxylase broths were inoculated with two to four colonies picked from a pure culture plate and overlaid with sterile mineral oil. They were incubated at $37^{\circ} \mathrm{C}$ and checked at half-hour intervals for up to eight hours. Final readings were made after 24 hours' incubation.

Møeller's decarboxylase broth with $1.0 \%$ lysine, arginine, or ornithine was purchased commercially (BBL). Each broth was inoculated with two to four colonies, overlaid with sterile mineral oil and incubated at $37^{\circ} \mathrm{C}$. Decarboxylase media with $0.3 \%$ agar was also purchased commercially (Hyland). These tubes were stab inoculated and incubated at $37^{\circ} \mathrm{C}$. Both Møller (1955) and agar decarboxylases were checked at hourly intervals for up to eight hours. Final readings were made after 24 hours' incubation.

The 479 Enterobacteriaceae were all tested in the rapid decarboxylase broth: 234 in parallel with Møller's broth and 245 in parallel with the agar base media (table IIA). Similarly, 286 non-fermentative Gram-negative rods were tested by the rapid method: 140 in parallel with Møller's broth and 146 in parallel with the agar base (table IIB).

If after 24 hours' incubation, the two methods being tested gave differing results with any isolate, all three decarboxylase reactions were retested with each method. If the repeat testing yielded identical results with both methods, it was assumed the initial different results(s) was due to an error in the inoculation procedure or a defect in a particular tube of medium. If, however, on repeat testing, the results obtained were the same as the original set of results, then the difference was recorded as a true difference between the two methods.

\begin{tabular}{lcc}
\hline Organism & Rapid vs Moller & Rapid vs Agar \\
\hline E. coli & 70 & 58 \\
K. pneumoniae & 62 & 79 \\
$P$. mirabilis & 32 & 36 \\
$P$. morganii & 5 & 3 \\
Citrobacter & 5 & 6 \\
$P$. stuartii & 1 & 0 \\
Serratia & 21 & 23 \\
E. aerogenes & 15 & 16 \\
E. cloacae & 21 & 23 \\
Aeromonas sp & 2 & 1 \\
Total & 234 & 245 \\
\hline
\end{tabular}

Table IIA Fresh clinical isolates of Enterobacteriaceae and Aeromonas used in the study

\begin{tabular}{lcc}
\hline Organism & Rapid vs Møller & Rapid vs Agar \\
\hline Herellea vaginicola & 56 & 44 \\
Mima polymorpha & 5 & 7 \\
P. aeruginosa & 58 & 75 \\
Alcaligenes sp & 2 & 3 \\
Flavobacterium sp & 2 & 3 \\
P. stutzeri & 3 & 3 \\
P. diminuta & 2 & 3 \\
P. putida & 3 & 2 \\
$P$. cepacia & 2 & 1 \\
P. maltophilia & 4 & 2 \\
P. alcaligenes & 3 & 2 \\
Moraxella sp & 0 & 1 \\
Total & 140 & 146 \\
\hline
\end{tabular}

Table IIB Non-fermentative Gram-negative bacilli

\section{Results}

The results of the comparative studies with Enterobacteriaceae are presented in table III. The rapid method appears more sensitive in detecting decarboxylase and dihydrolase activity after a 24-hour incubation than either the Møller method or the agar method. Both the later methods are similar in their comparison with the rapid method, and neither appears to be superior to the other. The rapid method detected arginine decarboxylase and dihydrolase 13 times more than the Møller method 


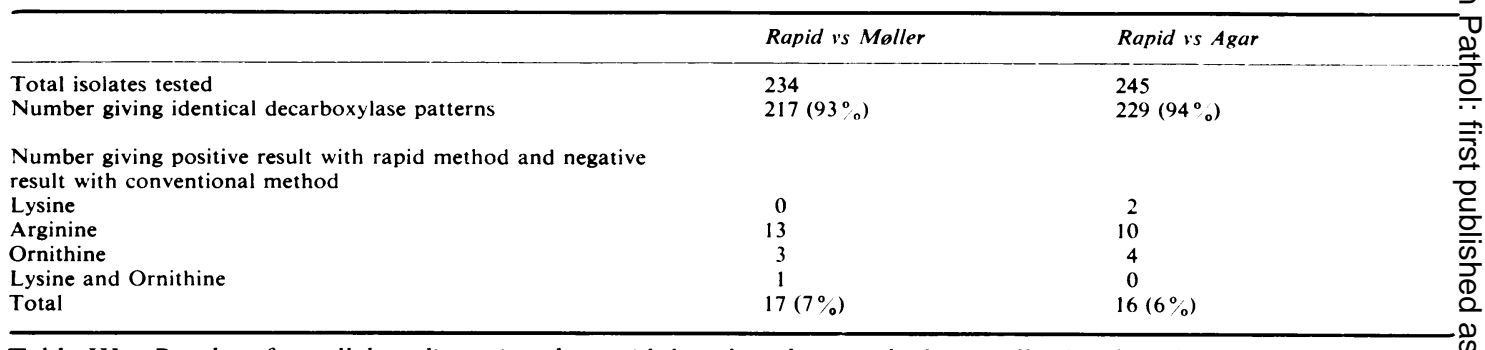

Table III Results of parallel studies using the rapid decarboxylase method vs Moller broth and agar decarboxylase for resting Enterobacteriaceae

and 10 times more than the agar method. Lysine and ornithine were also detected more frequently by the rapid method. In no instance did the conventional methods detect a positive decarboxylase or dihydrolase which was not also detected by the rapid method.

Table IV gives a breakdown according to isolates which gave positive reactions with the rapid method and negative reactions with the conventional methods. The 17 isolates of $E$. coli are perhaps the least impressive, as normally decarboxylase reactions are not needed for identification of this species. Positive arginines for Aeromonas, Citrobacter, and E. cloacae, as well as a positive lysine for Klebsiella are widely used in identification schemes.

The results of the parallel studies using nonfermentative Gram-negative bacilli are presented in table $\mathrm{V}$. When the rapid method was tested against the Møller method, all three isolates which gave positive results with the rapid but not the Møller method were strains of Pseudomonas aeruginosa.
In our study four strains of $P$. aeruginosa were arginine and ornithine positive. Gilardi (1971 an 1972), using Møller decarboxylase broth, found nov strains of $P$. aeruginosa which produced ornithineiv decarboxylase. Richard (1968), on the other hand $\vec{P}$ has also found strains of $P$. aeruginosa which are ornithine positive, using a medium similar to the one described by Fay and Barry (1972) plus $0 \cdot 1 \%$ glucose. In testing the rapid method against the agar method,, $\mathbb{D}$ one strain of $P$. aeruginosa and one strain of $P$. maltophilia gave positive results with the former method but not with the latter.

Although the final readings in all experiments $\vec{\theta}$ were made after 24 hours' incubation, the rap ${ }^{+}$ method was monitored at half-hour intervals up fo응 eight hours and the Moller and agar methods wefes monitored at hourly intervals up to eight hours. In looking at the rapid method, ornithine decarboxylase was detected as quickly as one hour after $\stackrel{\mathbb{Q}}{\varrho}$ inoculation, but was generally detected after two to $\Rightarrow$

\begin{tabular}{|c|c|c|c|c|c|c|}
\hline \multirow[t]{2}{*}{ Organism } & \multicolumn{3}{|c|}{ Rapid is Moller } & \multicolumn{3}{|c|}{ Rapid is Agar } \\
\hline & Lysine & Argirine & Orthinine & Lysine & Arginine & Orthinine \\
\hline Aeromonas & $\ldots$ & 2 & $\ldots$ & - & - & - \\
\hline Citrobacter & - & 2 & - & $\ldots$ & 4 & - \\
\hline E. cloacae & - & 2 &.-- & - & 5 & - \\
\hline E. coli & 1 & $\overline{7}$ & 4 & 1 & 1 & 4 \\
\hline K. pneumoniae & - & - & - & 1 & - & - \\
\hline
\end{tabular}

Table IV Breakdown of differing results by organism ${ }^{1}$

'Organisms listed gave positive res $\lrcorner$ lts with rapid method and negative results with conventional method

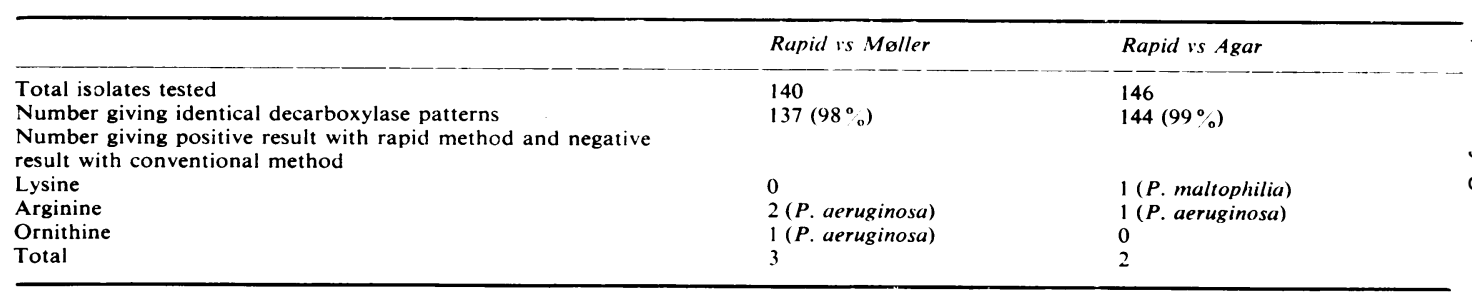

Table V Results of parallel studies using the rapid decarboxylase method vs Moller broth and agar decarboxylase for testing nonfermentative Gram-negative bacilli 
four hours' incubation. These findings confirm those of Fay and Barry (1972). The activity of lysine decarboxylase was detected at time intervals similar to ornithine. Arginine decarboxylase and dihydrolase were detected more slowly. Generally, it could be detected at six to eight hours but occasionally overnight incubation was required. In this particular study, if cultures were not positive at the end of the eight-hour day, they were simply incubated overnight, with the final reading being recorded after 24 hours' incubation. With both the Møller and agar methods lysine and ornithine decarboxylases were occasionally detected after seven to eight hours' incubation but generally required overnight incubation. Arginine always required overnight incubation for positive reactions.

All rapid and Møller media were incubated for an additional 24 hours and read for delayed positive reactions. The agar medium cannot be incubated for more than 24 hours (Fay and Barry, 1972). The rapid method yielded no positive reactions at the end of 48 hours which were not positive after 24 hours. However, Møller methods yielded numerous new positive reactions after the additional 24 hours' incubation, all of which had been positive with the rapid method after the initial 24 hours' incubation. Presumably, even more positives would have been noted had the cultures been incubated for four days (Møller, 1955).

\section{Discussion}

In conventional decarboxylase and dihydrolase tests, the organism initially ferments glucose in the medium which lowers the $\mathrm{pH}$ to the optimal hydrogen ion concentration for decarboxylase activity and changes the bromocresol purple indicator from purple to yellow. As decarboxylation or dihydrolation takes place, amines are released which raise the $\mathrm{pH}$ and revert the indicator back to its original purple colour. A control tube containing glucose but no amino acid is generally inoculated to insure that the test organism is a glucose fermenter. This later characteristic can be determined by a variety of media. In the present study a triple sugar iron agar slant was used to separate the glucose fermenters and nonfermenters and thus the decarboxylase control tube could be eliminated. In the rapid method glucose was not added to the media, thus eliminating the need for a control tube. The initial $\mathrm{pH}$ of the rapid media was lowered to $5 \cdot 0-5 \cdot 5$ which caused the indicator to turn a definite yellow colour. This pH was also optimal for decarboxylase and dihydrolase activity. These changes incorporated into the rapid method are essential in enabling decarboxy- lase and dihydrolase activity to be detected more quickly than by conventional means, as the organisms are able to attack the amino acids immediately, without first fermenting the glucose to obtain an optimal $\mathrm{pH}$ for this activity.

From the results of this study it is evident that the rapid method can detect lysine, arginine, and ornithine decarboxylase activity and arginine dihydrolase activity more rapidly than either of the two conventional methods, namely, the Møller and the $0.3 \%$ agar methods. Strong reactions, such as those seen after 24 hours' incubation, are of diagnostic value in distinguishing various genera and species of bacteria (Møller, 1955). Reactions normally detected after 24 hours' incubation of the agar medium and after 48 hours' incubation of the Møller broth can routinely be detected in two to eight hours with the rapid methods. As was mentioned earlier, an occasional strain will require overnight incubation with the rapid method, but these are generally strains requiring 48 hours' incubation or more in Møller broth.

Rarely a strain of $E$. coli reduced the bromcresol purple indicator giving a light gray or violet colour in the decarboxylase broth. This has also been noted by previous investigators (Fay and Barry, 1972; Møller, 1955). Indicator reduction was not hard to distinguish from a true positive purple colour and was not seen with other species of Enterobacteriaceae. It was noted more regularly among the non-fermentative, Gram-negative bacilli. However, it presented very little difficulty in interpretation of results.

The question arises as to whether reducing the volume of Møller broth and agar medium from $5 \mathrm{ml}$ to $1 \mathrm{ml}$, as was used for the rapid broth, would shorten the time required for detection or positive reactions. Fay and Barry (1972) showed that neither reducing the $\mathrm{pH}$ of Møller broth and agar medium to 5.5 nor reducing the volume of medium to $1 \mathrm{ml}$ caused decarboxylation of dihydrolation to occur in a shorter time.

On the basis of these results the rapid method for determining the decarboxylase activity of lysine and ornithine and the decarboxylase and dihydrolase activity of arginine is recommended for use in the routine clinical laboratory. The rapid method has proved comparable to the conventional methods in the results obtained, with the added advantage of shortened incubation times leading to quicker determination of results. Studies are currently in progress to determine whether or not the decarboxylase media can be overlaid with sterile mineral oil before autoclaving and then inoculated with the test organism directly through the oil into the medium. 
References

Blair, J. E., Lenette, E. H., and Truant, J. P., Editors (1970). In Manual of Clinical Microbiology. American Society for Microbiology, Bethesda, Maryland.

Edwards, P. R., and Ewing, W. H. (1972). Identification of Enterobacteriaceae, 3rd ed. Burgess, Minneapolis, Minnesota.

Fav, G. D., and Barry, A. L. (1972). Rapid ornithine decarboxylase test for the identification of Enterobacteriaceae. Appl. Microbiol., 23, 710-713.

Gilardi, G. L. (1971). Characterization of Pseudomonas species isolated from clinical specimens. Appl. Microbio!., 21, 414-419.
Gilardi, G. L. (1972). Practical scheme for the identification of non- קספ fermentative gram negative bacteria encountered in medical bacteriology. Amer. J. med. Technol., 38, 65-72.

Goldschmidt, M. C. Lockhart, B. M., and Perry, K. (1971). Rapid methods for determining decarboxylase activity: ornithine and lysine decarboxylases. Appl. Microbio ., 22, 344-349.

Møller, V. (1955). Simplified tests for some amıno acid decarboxy lases and for the arginine dihydrolase system. Acta path. microbiol. scand., 36, 158-172.

Richard, C. (1968). Techniques rapides de recherche des lysine $\frac{-\overline{6}}{-}$ décarboxylase, ornithine-décarboxylase et arginine-dihydrolase dans les genres Pseudomonas Alcaligenes, et Moraxella. 4nn. Inst. Pasteur (Paris), 114, 425-430.

\section{Reports and Bulletins prepared by the Association of Clinical Biochemists $\overrightarrow{\vec{\omega}}$}

The following reports and bulletins are published by the Association of Clinical Biochemists. They may be obtainedo․ from Dr D. H. Orrell, Department of Pathology, Royal Infirmary, Chorley New Road, Bolton BL1 4QS. The prices include postage, but air mail will be charged extra. Overseas readers should remit by British Postal or Money Order. If this is not possible the equivalent of $50 \mathrm{p}$ is the minimum amount that can be accepted.

SCIENTIFIC REPORTS

3 Automatic Dispensing Pipettes: an assessment of 35 commercial instruments September 1967 P. M. G. BROUGHTON, A. H. GOWENLOCK, G. M. WIDDOWSON, and K. A. AHLQUIST $80 \mathrm{p}(\$ 2)$

4 An Evaluation of five Commercial Flame Photometers suitable for the Simultaneous Determination of Sodium and Potassium March 1970 P. M. G. BROUGHTON and J. B. DAWSON $80 \mathrm{p}(\$ 2)$

\section{SCIENTIFIC REVIEWS}

1 The Assessment of Thyroid Function March 1971 F. V. FLYNN and J. R. HOBBS $60 \mathrm{p}(\$ 1.50)$

2 Renal Function Tests Suitable for Clinical Practice January 1972 F. L. MITCHELL, N. VEALL, and R. W. E. WATTS $60 \mathrm{p}(\$ 1.50)$

\section{TECHNICAL BULLETINS}

9 Determination of Urea by AutoAnalyzer November 1966 RUTH M. HASLAM 40p (\$1)

11 Determination of Serum Albumin by AutoAnalyzer using Bromocresol Green October 1967 B. E. NORTHAM and G. M. WIDDOwson $40 \mathrm{p}(\$ 1)$

13 An Assessment of the Technicon Type II Sampler Unit March 1968 B. C. GRAY and G. K. MCGOWAN $40 \mathrm{p}(\$ 1)$

14 Atomic Absorption Spectroscopy: an outline of its principles and a guide to the selection of instruments May 1968 J. B. DAWSON and P. M. G. BROUGHTON $40 \mathrm{p}(\$ 1)$

15 A Guide to Automatic Pipettes (2nd edition) June 1968 P. M. G. BROUGHTON 40p (\$1)

16 A Guide to Automation in Clinical Chemistry May 1969 P. M. G. BROUGHTON 60p (\$1.50)

17 Flame Photometers: a comparative list of 17 instruments readily available in Britain August 1969 P. WILDING $60 \mathrm{p}(\$ 1.50)$
19 Spectrophotometers. A comparative list of low-priced $\overrightarrow{\oplus 0}$ instruments readily available in Britain May $1970 \mathrm{~b}$ C. E. WILDE and P. SEWELL $60 \mathrm{p}(\$ 1.50)$

20 Quantities and Units in Clinical Biochemistry Juneep 1970 P. M. G. BROUGHTON 60p (\$1.50) More than 30 copies in units of 10 at $20 \mathrm{p}$ each

21 Filter Fluorimeters: A comparative list of 18 instru $\overrightarrow{0}$

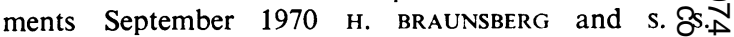
BROWN $60 \mathrm{p}(\$ 1.50)$

22 Bilirubin Standards and the Determination of Biliru望n by Manual and Technicon AutoAnalyzer Methodso January 1971 BARBARA BILLING, RUTH HASLAM, anç̋ N. WALD $60 \mathrm{p}(\$ 1.50)$

23 Interchangeable Cells for Spectrophotometers anch Fluorimeters September 1971 S. S. BROWN and A. $\mathrm{H}_{5}$ GOWENLOCK $60 \mathrm{p}(\$ 1.50)$

24 Simple Tests to Detect Poisons March 1972 B. wत् MEADE et al. $60 \mathrm{p}(\$ 1.50)$

25 Blood Gas Analysers May 1972 K. DIXON 60p $(\$ 1.50)$

26 Kits for Enzyme Activity Determination Septembeㅁำ 1972 S. B. ROSALKI and D. TARLOW 80p $(\$ 2.00)$

27 Assessment of Pumps Suitable for Incorporation intō Existing Continuous Flow Analytical Systems November 1972 A. FLECK et al. $60 \mathrm{p}(\$ 1.50)$

28 Routine Clinical Measurements of Transferrin $\omega$ Human Serum September 1973 K. DIXON 60p $(\$ 1.50$ )

29 Control Materials for Clinical Biochemistry (5t) edition) September 1973 J. F. STEVENS 60p $(\$ 1.50)$

30 Notes on the Quality of Performance of Serun? Cholesterol Assays September 1973 s. S. BROWN 60Q $(\$ 1.50)$ 